LIVER

\title{
Prevalence and clinical significance of isotype specific antinuclear antibodies in primary biliary cirrhosis
}

\author{
E I Rigopoulou, E T Davies, A Pares, K Zachou, C Liaskos, D-P Bogdanos, J Rodes, G N Dalekos, \\ D Vergani
}

Gut 2005;54:528-532. doi: 10.1136/gut.2003.036558

See end of article for authors' affiliations

Correspondence to: Professor D Vergani Institute of Liver Studies, King's College Hospital Denmark Hill, London SE5 9RS, UK; diego.vergani@ kcl.ac.uk

Revised version received 8 October 2004 Accepted for publication 11 October 2004

\begin{abstract}
Background: Antinuclear antibodies (ANA) giving a rim-like/membranous (RL/M) or a multiple nuclear dot (MND) pattern are highly specific for primary biliary cirrhosis (PBC).

Aim and subjects: To assess the prevalence of $\mathrm{PBC}$ specific ANAs, their $\mathrm{lg}$ isotype, and their clinical significance in 90 PBC patients from Greece and Spain. Twenty eight patients with chronic hepatitis C, 23 patients with systemic lupus erythematosus, and 17 healthy subjects were studied as controls.

Methods: PBC specific ANA reactivity was tested by indirect immunofluorescence using HEp2 cells as substrate and individual $\lg$ class (lgG, $\lg A, \lg M)$ and $\lg G$ subclass (lgG1, $\lg G 2, \lg G 3, \lg G 4)$ specific antisera as revealing reagents.

Results: Fourteen of 90 (15.6\%) PBC patients had PBC specific ANA reactivity when an anti-lgG (total) antiserum was used as the revealing reagent while $58(64.4 \%)$ were positive when specific antisera to each of the four lgG isotypes were used. The prevailing isotype was $\lg G 3$ for MND and $\lg G 1$ for RL/M. PBC patients with specific ANA, in particular of the lgG3 isotype, had significantly more severe biochemical and histological disease compared with those who were seronegative. None of the controls was positive. Conclusions: Disease specific ANA are present in the majority of patients with PBC when investigated at the level of immunoglobulin isotype. PBC specific ANA, in particular of the lgG3 isotype, are associated with a more severe disease course, possibly reflecting the peculiar ability of this isotype to engage mediators of damage.
\end{abstract}

$\mathrm{P}$ rimary biliary cirrhosis (PBC) is an immune mediated chronic cholestatic liver disease that affects mainly middle aged women and is characterised by the progressive destruction of the small intrahepatic bile ducts leading to cirrhosis. ${ }^{1}$ The characteristic immunological feature of the disease is the presence of circulating antimitochondrial antibodies (AMA) directed against components (collectively named M2) of the inner mitochondrial multienzyme 2-oxoacid dehydrogenase complexes. ${ }^{2}$ Although AMA are considered to be the hallmark of PBC, they are not the only disease specific autoantibodies. ${ }^{2}{ }^{3}$ Previous studies have shown that two other sets of antibodies against nuclear antigens (ANA) giving a multiple nuclear dot (MND) ${ }^{4}$ and a rim-like/membranous (RL/M) $)^{5}$ pattern by indirect immunofluorescence (IIFL) are specific for PBC, are found in up to $50 \%$ of patients with this condition, ${ }^{6-14}$ and may have prognostic significance. ${ }^{15-18}$ These reports evaluated individual PBC specific ANA specificities, the exception being a recent study by Muratori and colleagues ${ }^{18}$ who investigated several ANA reactivities. No histological correlates were however presented in that work. None of these studies investigated the immunoglobulin (Ig) isotype of PBC specific ANAs.

The aim of the present study was to assess the prevalence of a panel of PBC specific ANAs, their Ig isotype, and their clinical significance in patients with PBC. In view of the reported geographical diversity of PBC manifestations, ${ }^{19-22}$ we enrolled two groups of patients, 55 from Greece and 35 from Spain.

\section{MATERIALS AND METHODS PBC patients}

Serum samples from 90 patients with $\mathrm{PBC},{ }^{1}$ including 55 Greek and 35 Spanish patients, were studied. All Greek patients were followed up at the outpatient clinic of the Academic Liver Unit, Department of Internal Medicine, University Hospital of Larissa, Thessaly, Central Greece. All Spanish patients were followed up at the Liver Unit, Hospital Clinic, University of Barcelona, Spain. At the time of serum sample collection all but five patients (three Greek and two Spanish) were AMA positive by IIFL (median titre 1/640 (range $1 / 40-1 / 5120)$ ). All but two patients had a liver biopsy available for review. According to Ludwig's histological classification, ${ }^{23} 30$ patients had stage I (20 Greek), 17 stage II (six Greek), 20 stage III (12 Greek), and 21 stage IV PBC (15 Greek). Three patients were classified as PBC-autoimmune hepatitis overlap syndrome. ${ }^{24}$ Application of the revised scoring system of the International Autoimmune Hepatitis Group $^{25}$ excluded the diagnosis of probable or definitive autoimmune hepatitis in the remaining 88 patients (score $<10$ ). Thirty two patients were studied at diagnosis, before administration of ursodeoxycholic acid (UDCA). The Mayo risk score ${ }^{26}$ was calculated as a prognostic index in all patients. Based on their clinical, biochemical, and histological features, 21 patients (15 Greek and six Spanish) were classified as cirrhotic. Fourteen of the 90 patients died during follow up; 10 from liver related complications (seven Greek and three Spanish). Four patients received a liver transplant.

Presence of clinical signs and symptoms was assessed at presentation and monitored during follow up visits, including jaundice, fatigue, pruritus, and major complications of cirrhosis, such as variceal bleeding, ascites, infection, and hepatic encephalopathy.

\footnotetext{
Abbreviations: $\mathrm{AMA}$, antimitochondrial antibody; ANA, antinuclear antibody; $\mathrm{HCV}$, hepatitis $\mathrm{C}$ virus; Ig, immunoglobulin; IIFL, indirect immunofluorescence; MND, multiple nuclear dot; $\mathrm{OR}$, odds ratio; $\mathrm{PBC}$ primary biliary cirrhosis; RL/M, rim-like/membranous; UDCA, ursodeoxycholic acid
} 


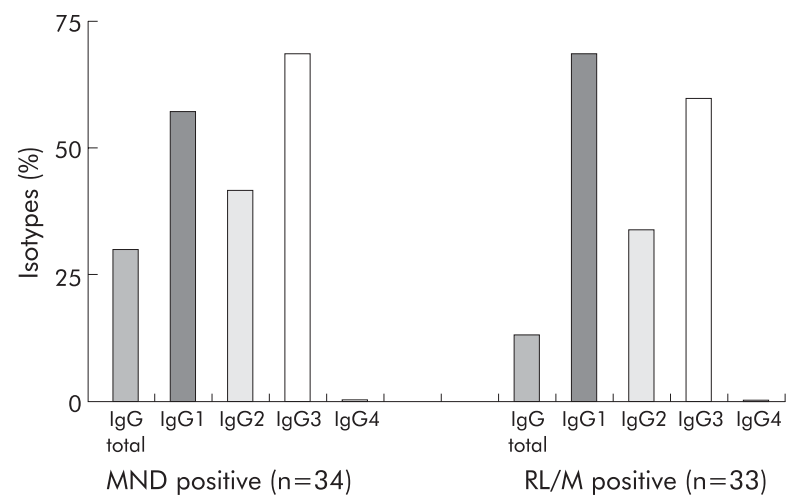

Figure 1 Immunoglobulin G (lgG) isotype of multiple nuclear dot (MND) and rim-like/membranous (RL/M) autoantibodies in patients with primary biliary cirrhosis expressed as a percentage. The distribution of the autoantibody isotypes differed from that of normal serum lgG which followed the $\operatorname{lgG} 1>\lg G 2>\lg G 3>\lg G 4$ pattern. More detailed data are available in appendix 1 on the Gut website at http:// www.gut.com/supplemental.

Other causes of cholestasis, infection with hepatitis B and $\mathrm{C}$ virus, human immunodeficiency virus, Wilson disease, and haemochromatosis were excluded. Liver function tests, urea, creatinine, prothrombin time, and immunoglobulins were measured by routine methods.

\section{Pathological and normal controls}

Twenty eight patients with chronic hepatitis C virus (HCV) infection (mean age 56.2 (17.4) years, 25 female), all HCV RNA positive, and 23 patients with systemic lupus erythematosus (mean age 36.2 (18.1) years, 20 female) were tested as pathological controls; 17 healthy volunteer members of staff (mean age 47.2 (17.4) years, 16 female) were tested as normal controls.

\section{Autoantibody detection}

Presence of AMA and ANA was initially detected by conventional IIFL on $5 \mu \mathrm{m}$ frozen sections of a composite substrate containing rat liver, kidney, and stomach using, as revealing reagent, an anti-total human Ig-fluorescein isothiocyanate conjugate (IgG, A, M, $\kappa$ and $\lambda$; Dako Ltd, High Wycombe, Bucks, UK). ${ }^{27-29}$ Serum samples were screened at a dilution of 1/40 in accordance with our laboratory practice. AMA reactivity was further evaluated by immunoblot and ELISA using an M2 antigen purified from porcine heart mitochondria (Pharmacia and Upjohn, Milton Keynes, UK). ${ }^{3031}$

To better define the ANA pattern, all sera were also evaluated using commercially available human epithelial cells (HEp-2010; Euroimmun, Cardiff, UK). This line was generated from HEp2 cells to improve the detection of the nuclear dots pattern, which on this subline gives a dense coarse-granular fluorescence; the HEp-2010 line also allows detection of all other ANA specificities. All serum samples were tested at a starting dilution of $1 / 40$. The second reagent comprised fluorescein labelled rabbit antisera specifically targeting IgG, IgA, or IgM (Dako) and used at a dilution of $1 / 50$ in phosphate buffered saline. Affinity purified fluorescein labelled sheep antihuman IgG subclass antisera (Gl, G2, G3, G4; Binding Site, Birmingham, UK) diluted to $1 / 50$ in phosphate buffered saline were also used in another set of experiments. Each slide was examined by an independent observer (ETD) using an Olympus BX40 (Olympus Optical, London, UK) fluorescence microscope.

All subjects consented to participate in the study. The local ethical committees approved the study protocol.

\section{Statistical analysis}

Data are presented as percentages (\%) or mean (SD), unless otherwise stated. Differences between autoantibody positive and negative patients for various clinical features, histological features, and serological measurements were compared using the $t$ test, the Mann Whitney U test, $\chi^{2}$ test (two by two with Yates' correction), or Fisher's exact test, as appropriate. Logistic regression analysis was performed for multiple comparisons. Two sided $\mathrm{p}$ values $<0.05$ were considered statistically significant. Statistical analyses were performed using the SPSS (SPSS Inc., version 10.0, Chicago, Illinois, USA) statistical package.

Table 1 Comparison of clinical, biochemical, and histological features of 90 Greek and Spanish patients with primary biliary cirrhosis (PBC) according to multiple nuclear dots (MND) and/or rim-like membranous pattern (RL/M) reactivity

\begin{tabular}{|c|c|c|c|c|c|c|c|c|c|}
\hline & $\begin{array}{l}\text { Total PBC } \\
(n=90)\end{array}$ & $\begin{array}{l}\text { Positive PBC } \\
\text { specific ANA } \\
(n=65)\end{array}$ & $\begin{array}{l}\text { Negative PBC } \\
\text { specific ANA } \\
(n=25)\end{array}$ & $\begin{array}{l}\text { Positive } \\
\text { MND } \\
(n=37)\end{array}$ & $\begin{array}{l}\text { Negative } \\
\text { MND } \\
(n=53)\end{array}$ & $\begin{array}{l}\text { Positive } \\
\text { RL/M } \\
\text { (n=42) }\end{array}$ & $\begin{array}{l}\text { Negative } \\
\mathrm{RL} / \mathrm{M} \\
(\mathrm{n}=48)\end{array}$ & $\begin{array}{l}\text { Positive PBC } \\
\text { specific ANA } \\
\text { lgG3 } \\
(n=42)\end{array}$ & $\begin{array}{l}\text { Negative PBC } \\
\text { specific ANA } \\
\text { lgG3 } \\
(n=48)\end{array}$ \\
\hline Age (y) & $58(11.8)$ & $58(11.5)$ & $58(13)$ & $58(12.5)$ & $58(11.5)$ & $60(10.2)$ & 57 (13) & $59(9.5)$ & $57(13.6)$ \\
\hline Duration of disease (months) & $48(61.6)$ & $52(50)$ & $37(55)$ & $59(54)^{*}$ & $40(49)^{*}$ & $49(53)$ & $47(51)$ & $66(52.5)^{\star * * *}$ & $32(45.7)^{* * \star *}$ \\
\hline Bilirubin (mg/dl) & $1.3(1.1)$ & $1.4(1.2)$ & $1(0.6)$ & $1.5(1.2)$ & $1.1(1)$ & $1.5(1.4)$ & $1.1(0.8)$ & $1.5(1.3)$ & $1.1(0.8)$ \\
\hline Albumin (g/dl) & $4.1(0.6)$ & $4(0.6)$ & $4.3(0.5)$ & $4(0.6)^{*}$ & $4.2(0.6)^{*}$ & $4(0.7)$ & $4.2(0.5)$ & $4(0.6)^{*}$ & $4.2(0.6)^{*}$ \\
\hline AST (U/I) (nv <40) & 59 (67) & $63(76)$ & $48(27)$ & $70(96)$ & $60(51)$ & $71(94)$ & $48(25)$ & $68(92)$ & $51(30)$ \\
\hline ALT (U/I) (nv < 40) & $66(62)$ & $69(68)$ & $58(45)$ & $74(75)$ & $60(51)$ & $75(79)$ & $58(42)$ & $67(71)$ & $65(54)$ \\
\hline $\operatorname{ALP}(U / \mathrm{I})(\mathrm{nv}<104)$ & 339 (313) & $318(290)$ & $394(366)$ & $310(237)$ & $360(357)$ & $323(335)$ & $353(295)$ & $375(330)$ & $308(297)$ \\
\hline$\gamma \mathrm{GT}(\mathrm{U} / \mathrm{I})(\mathrm{nv}<50)$ & $201(190)$ & 187 (183) & 235 (207) & $188(173)$ & 209 (202) & $182(182)$ & $216(197)$ & $214(204)$ & $189(179)$ \\
\hline $\operatorname{lgG}(\mathrm{mg} / \mathrm{dl})(\mathrm{nv}<1690)$ & $1666(558)$ & $1677(561)$ & 1637 (559) & $1758(671)$ & $1600(456)$ & $1635(490)$ & $1692(614)$ & $1772(593)^{*}$ & $1571(511)^{*}$ \\
\hline $\lg M(\mathrm{mg} / \mathrm{dl})(\mathrm{nv}<249)$ & $387(249)$ & $390(251)$ & $379(252)$ & $394(269)$ & 382 (237) & $385(206)$ & $389(284)$ & $398(222)$ & $377(273)$ \\
\hline $\lg A(\mathrm{mg} / \mathrm{dl})(\mathrm{nv}<300)$ & $310(148)$ & $305(141)$ & $324(170)$ & 307 (143) & 312 (153) & 311 (151) & 309 (148) & 320 (134) & 301 (161) \\
\hline Creatinine $(\mathrm{mg} / \mathrm{dl})$ & $0.9(0.4)$ & $0.9(0.4)$ & $0.8(0.2)$ & $0.9(0.4)$ & $0.8(0.3)$ & $0.9(0.4)$ & $0.9(0.3)$ & $0.9(0.5)$ & $0.8(0.2)$ \\
\hline Mayo risk score & $4.9(1.5)$ & $5(1.6)$ & $4.5(0.9)$ & $5.2(1.8)$ & $4.6(1.1)$ & $5.2(1.7)$ & $4.6(1.2)$ & $5.2(1.7)^{*}$ & $4.6(1.2)^{*}$ \\
\hline Histological stage & $2.4(1.2)$ & $2.6(1.2)^{* * * *}$ & $1.8(0.9)^{* * *}$ & $2.7(1.2)^{*}$ & $2.1(1.1)^{*}$ & $2.7(1.1)^{* *}$ & $2.1(1.2)^{* *}$ & $2.7(1.1)^{\star * *}$ & $2(1.1)^{\star * *}$ \\
\hline Histological stage (I-III/IV) & $68 / 20$ & $45 / 19^{* * * *}$ & $23 / 1^{* * * *}$ & $22 / 14^{* * *}$ & $46 / 6^{* * *}$ & $29 / 12$ & $39 / 8$ & $28 / 14^{*}$ & $39 / 7^{*}$ \\
\hline Non cirrhotic/cirrhotic & $69 / 21$ & $45 / 20^{\star \star \star \star}$ & $24 / 1^{* * * *}$ & $22 / 15^{\star \star * *}$ & $47 / 6^{* * * *}$ & $30 / 12$ & $39 / 9$ & $28 / 14^{*}$ & $41 / 7^{*}$ \\
\hline $\begin{array}{l}\text { Liver related deaths or } \\
\text { transplantation }(\mathrm{n} / \mathrm{y})\end{array}$ & $76 / 14$ & $52 / 13^{*}$ & $24 / 1^{*}$ & $26 / 11^{* * * *}$ & $50 / 3^{* * * *}$ & $35 / 7$ & $41 / 7$ & $32 / 10^{*}$ & $44 / 4^{*}$ \\
\hline \multicolumn{10}{|c|}{$\begin{array}{l}\text { Data are expressed as mean }(S D) \\
{ }^{*} 0.1>p>0.05 ;{ }^{* *} p \leqslant 0.05 ;{ }^{* \star} p \leqslant 0.01 ;{ }^{* * * *} p \leqslant 0.005 \text {. } \\
\text { AST, aspartate aminotransferase; ALT, alanine aminotransferase; ALP, alkaline phosphatase, } \gamma \mathrm{GT} \text {, gamma glutamyl transpeptidase; UDCA, ursodeoxycholic acid } \\
n v \text {, normal values; } n \text {, no; } y \text {, yes. }\end{array}$} \\
\hline
\end{tabular}



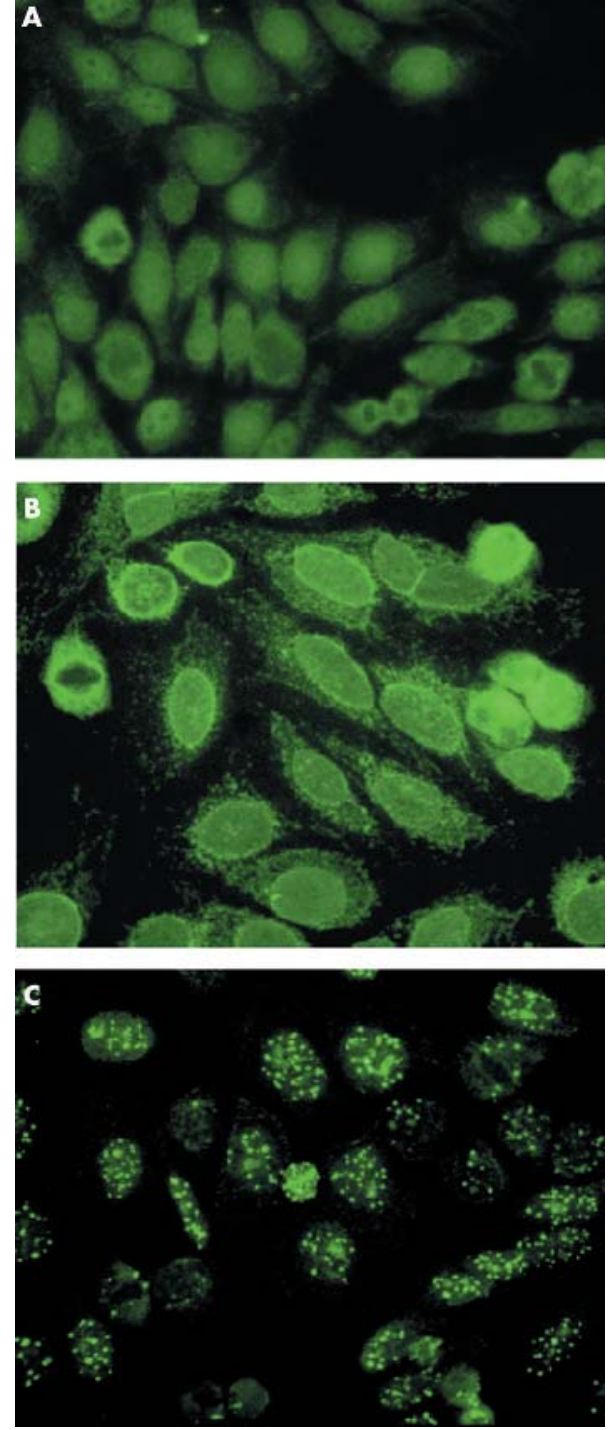

Figure 2 Comparison of patterns obtained with anti-lgG total and specific anti-isotype $G$ antisera (lgGl , 2, 3, 4) on a representative case by indirect immunofluorescence on HEp-2010 cell substrate. The use of anti-total lgG gave a negative result (A) while anti-lgGl gave a rim-like membranous pattern (B) and anti-lgG3 a multiple nuclear dot pattern (C).

\section{RESULTS}

\section{Prevalence of PBC specific ANA}

Fourteen of 90 (15.6\%) PBC patients had PBC specific ANA reactivity ( $10 \mathrm{MND}$ and four RL/M) when an anti-IgG (total) antiserum was used as the revealing reagent while 58 $(64.4 \%)$ were positive when specific antisera to each of the four IgG isotypes were used. An additional seven were positive for IgM alone.

MND positivity was present in 37 of 90 (41.1\%) patients; 34 had positivity for IgG isotypes, belonging to IgGl in 19 (55.9\%), IgG2 in 15 (44.1\%), IgG3 in $23(67.6 \%)$, and IgG4 in none (fig 1). More detailed data are available in appendix 1 which can be viewed on the Gut website at http://www.gut. com/supplemental.

RL/M positivity was present in 42 of 90 (46.7\%) patients; $33 \mathrm{had} \mathrm{RL} / \mathrm{M}$ positivity for IgG isotypes, belonging to IgGl in $22(66.7 \%), \operatorname{IgG} 2$ in 11 (33.3\%), IgG3 in $19(57.6 \%)$, and IgG4 in none (fig 1). Of the 65 samples positive for $\mathrm{PBC}$ specific ANA on IIFL at a dilution of $1 / 40,51(78 \%)$ reacted at $1 / 160$ and $37(57 \%)$ at a dilution of $1 / 640$.

\section{Clinical significance of PBC specific ANA}

Anti-MND positive patients of any isotype had significantly more severe liver disease than those anti-MND negative, as shown by the higher frequency of cirrhosis (odds ratio (OR) 0.19 (95\% confidence interval (CI) 0.06-0.5); $\mathrm{p}=0.003$ ) and worse outcome (OR 0.142 (95\% CI $0.04-0.6) ; p=0.003$ ) (table 1). Patients with RL/M had histologically more severe disease than those seronegative (95\% CI 0.06-1.04; $p=0.03$, $t$ test) (table 1 ).

PBC patients with MND, RL/M reactivity, or both—of any isotype-were characterised by histologically more severe disease (95\% CI $0.3-1.3 ; \mathrm{p}=0.003, t$ test), higher frequency of cirrhosis (OR 0.09 (95\% CI 0.01-0.7); $\mathrm{p}=0.005$ ), and tended to have a worse outcome (OR 0.16 (95\% CI $0.02-1.3$ ); $\mathrm{p}=0.06)$ (table 1$)$.

Patients positive for both MND and RL/M-of any isotype-had more severe disease compared with patients positive for single ANA reactivities or with patients negative for any PBC specific ANA, as shown by histologically more advanced liver disease (95\% CI 0.44-1.8; $\mathrm{p}=0.002, t$ test), higher frequency of cirrhosis (OR 0.2 (95\% CI $0.07-0.7$ ); $\mathrm{p}=0.02)$, and worse outcome (OR $0.2(95 \% \mathrm{CI}-0.07-0.9$; $\mathrm{p}=0.04)($ table 1$)$.

Patients with ANA reactivity of the IgG3 subclass had histologically more severe disease (95\% CI 0.16-1.13; $\mathrm{p}=0.009, t$ test), higher frequency of cirrhosis (OR 0.3 $(95 \%$ CI $0.1-1) ; p=0.06$ ), worse outcome (OR 0.3 (95\% CI $0.08-1.01) ; \mathrm{p}=0.08$ ), and longer disease duration (95\% CI 13.4-54.5; $\mathrm{p}=0.002, t$ test), and were more frequently receiving UDCA (OR $1.2 \quad(95 \%$ CI $1.06-1.3 ; \quad \mathrm{p}=0.02)$ (table 1) than those IgG3 negative.

To assess whether the association with more severe histology was a reflection of a link to longer duration, 24 patients positive for IgG3 PBC specific ANA were case matched for disease duration to negative patients. Cirrhosis was more frequent in the first group of patients ( $11 / 24 v 1 / 24$; $\mathrm{p}=0.003)$.

Prevalence and clinical significance of PBC specific ANA within the Greek and Spanish populations were similar to those obtained in the undivided population.

None of the pathological and normal controls was found to be positive for any of the PBC specific ANA reactivities of any isotype.

\section{DISCUSSION}

Two major findings have emerged from the present study. Firstly, disease specific ANA are present in more than half of patients with PBC when investigated at the level of their individual immunoglobulin isotype. Secondly, PBC specific ANA, in particular those of the IgG3 isotype, can identify patients with a more severe disease and worse outcome.

Discrepancies between different studies reporting a 7-53\% prevalence of PBC specific ANA have been attributed either to the geographical diversity of the autoantibody profile in patients with $\mathrm{PBC}$ or to the difficulty in the interpretation of the results obtained by conventional IIFL. ${ }^{6-14}$ Clear visualisation of the RL/M or MND pattern is at times difficult when AMA and other antinuclear specificities are present in the same serum (see fig 2). ${ }^{45}$ Anti-total IgG antisera, routinely used for investigating PBC specific ANA, may detect suboptimally antibodies of IgG isotypes such as IgG3; this is due to the fact that IgG3 represents only $6 \%$ of the total IgG and antisera raised by administration to animals of total IgG sometimes fail to recognise this isotype. The same applies to IgG4 which represents an even smaller proportion of the total IgG $(2 \%) .^{32}$ Awareness of this is of particular importance in PBC where disease specific antibodies such as AMA have been shown to belong typically to the IgG3 subclass. ${ }^{33}$ 
Our results illustrate this point clearly: using a conventional anti-total IgG antiserum, we found ANA positivity in $15 \%$ of our patients, but when we used anti-IgG isotype specific antisera, seropositivity reached 65\%. Forty four patients positive for MND and/or RL/M would have gone undetected if we had only used the conventional anti-total IgG antiserum.

The use of antisera specific for individual isotypes therefore provides a more complete picture of the ANA prevalence in PBC and greatly reduces the limitations of conventional IIFL, as suggested by French and Bernstein ${ }^{34}$ in 1987. Enhanced sensitivity is frequently accompanied by a decrease in specificity. This does not appear to be the case in the present study as isotype specific RL/M or MND reactivities were absent in all controls that comprised healthy subjects, patients with chronic HCV infection, and patients with lupus. The latter observation is particularly important as positivity for ANA is the hallmark of systemic lupus erythematosus and none of the 23 lupus patients tested positive for PBC specific ANA.

Accurate detection of PBC specific ANA using IgG subclass specific antisera gave us the opportunity to reassess their clinical relevance. Contrary views as to whether PBC specific ANA are of prognostic or clinical importance have been expressed in previous studies using either conventional IIFL or molecularly based assays. ${ }^{6-14}$ 16-18 3536 Thus, Invernizzi and colleagues $^{17}$ studying reactivity against nuclear pore complex-the target of antibodies giving the RL/M immunofluorescent pattern-found that seropositive patients have cirrhosis and its complications more frequently than those seronegative. A subsequent study by Miyachi and colleagues ${ }^{35}$ was unable to substantiate these findings although it was based on similar antigenic preparations. Similarly, Itoh and colleagues $^{16}$ and Muratori and colleagues ${ }^{18}$ reported that the presence of antibodies to the nuclear envelope gp210 antigen is associated with disease severity and worse prognosis. However, no such link was found in earlier studies in larger groups of patients. ${ }^{7-9}$ These discrepancies may be due to differences in study design and to the variety of antigenic preparations used. In particular, a limitation of the molecular based assays is the need to select some targets, neglecting others of potential equal importance.

The advantage of the IgG isotype specific IIFL technique used in the present study is that, by its nature, it is able to detect all ANA specificities with the added bonus of being easy to perform.

Of clinical relevance, our own results indicate that patients positive for PBC specific ANA reactivities, of any of the Ig isotypes, have a histologically more advanced disease, are more frequently cirrhotic, and have a worse outcome, than those seronegative. Seropositivity for anti-MND, in particular, is associated with marked alterations in liver function tests, more advanced histological stage, presence of symptoms, and worse outcome.

ANA of the IgG3 class characterised patients with a longer disease duration and more severe histological picture. The possibility that the IgG3 isotype is associated with a more severe histology as a consequence of its association with longer duration of disease is refuted by the observation that in two groups of patients matched for disease duration, those with PBC specific ANA of IgG3 isotypes were more frequently cirrhotic. Longer duration of disease ${ }^{17}$ but similar age may suggest that the diagnosis of the disease in PBC specific ANA seropositive patients is made at an earlier time point compared with negative patients. Also, AMA have a substantial IgG3 component, and an elevated level of IgG3 anti-PDC-E2 is associated with more rapid disease progression. ${ }^{37}$ Peculiar to this immunoglobulin isotype is its ability to activate complement with high efficiency ${ }^{38}$ and to act as the principal mediator of antibody dependent cell mediated cytotoxicity, ${ }^{39}{ }^{40}$ the two main mechanisms through which antibodies inflict cell damage. ${ }^{41}$ Whether autoantibodies of the IgG3 isotype are of pathogenic relevance in $\mathrm{PBC}$ or just markers of disease activity, however, remains to be established in future prospective studies.

\section{ACKNOWLEDGEMENTS}

The authors thank Professor George Koukoulis for his evaluation of liver biopsies from patients with PBC, and Professor Giorgina MieliVergani for her critical comments. Dimitrios-Petrou Bogdanos is supported by the Children's Liver Disease Foundation, Birmingham, UK. This project was supported in part by a grant from the Instituto de Salud Carlos III (C03/02).

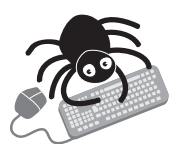

More detailed data on the clinical, biochemical, and histological features of the patients are available in appendix 1 which can be viewed on the Gut website at http://www.gut.com/supplemental.

\section{Authors' affiliations}

E I Rigopoulou*, K Zachou, C Liaskos, G N Dalekos, Academic Liver Unit and Research Laboratory of Internal Medicine, Department of Internal Medicine, Larissa Medical School, University of Thessaly, Larissa, Greece

E T Davies*, Department of Immunology, King's College, London, UK A Pares, J Rodes, Liver Unit, Institut de Malalties Digestives Hospital Clínic, IDIBAPS, University of Barcelona, Barcelona, Spain D-P Bogdanos, D Vergani, Institute of Liver Studies, King's College, London, UK

*E I Rigopoulou and E T Davies contributed equally to this work. Conflict of interest: None declared.

\section{REFERENCES}

1 Neuberger J. Primary biliary cirrhosis. Lancet 1997:350:875-9.

2 Bogdanos DP, Baum H, Vergani D. Antimitochondrial and other autoantibodies. Clin Liver Dis 2003;7:759-77.

3 Vergani D, Bogdanos DP. Positive markers in AMA-negative PBC. Am J Gastroenterol 2003;98:241-3.

4 Szostecki C, Guldner HH, Will H. Autoantibodies against "nuclear dots" in primary biliary cirrhosis. Semin Liver Dis 1997;17:71-8.

5 Courvalin JC, Worman HJ. Nuclear envelope protein autoantibodies in primary biliary cirrhosis. Semin Liver Dis 1997;17:79-90.

6 Bernstein RM, Neuberger JM, Bunn CC, et al. Diversity of autoantibodies in primary biliary cirrhosis and chronic active hepatitis. Clin Exp Immunol 1984;55:553-60.

7 Lozano F, Pares A, Borche L, et al. Autoantibodies against nuclear envelopeassociated proteins in primary biliary cirrhosis. Hepatology 1988;8:930-8.

8 Lassoued K, Brenard R, Degos F, et al. Antinuclear antibodies directed to a 200-kilodalton polypeptide of the nuclear envelope in primary biliary cirrhosis. A clinical and immunological study of a series of 150 patients with primary biliary cirrhosis. Gastroenterology 1990;99:181-6.

9 Nickowitz RE, Wozniak RW, Schaffner F, et al. Autoantibodies against integral membrane proteins of the nuclear envelope in patients with primary biliary cirrhosis. Gastroenterology 1994;106:193-9.

10 Powell F, Schroeter AL, Dickson ER. Antinuclear antibodies in primary biliary cirrhosis. Lancet 1984;1:288-9.

11 Shibata M, Ueno Y, Onozuka Y, et al. Autoantibodies against nuclear envelope in patients with primary biliary cirrhsosis. Internal Hepatol Comm, 1985;4:46-53.

12 Cassani $F$, Bianchi FB, Lenzi $M$, et al. Immunomorphological characterisation of antinuclear antibodies in chronic liver disease. J Clin Pathol 1985;38:801-5.

13 Ruffatti A, Arslan P, Floreani A, et al. Nuclear membrane-staining antinuclear antibody in patients with primary biliary cirrhosis. J Clin Immunol 1985;5:357-61.

14 Remmel T, Piirsoo A, Koiveer A, et al. Clinical significance of different antinuclear antibodies patterns in the course of primary biliary cirrhosis. Hepatogastroenterology 1996;43:1135-40.

15 Zuchner D, Sternsdorf T, Szostecki C, et al. Prevalence, kinetics, and therapeutic modulation of autoantibodies against Sp 100 and promyelocytic leukemia protein in a large cohort of patients with primary biliary cirrhosis. Hepatology 1997;26:1123-30.

16 Itoh S, Ichida T, Yoshida T, et al. Autoantibodies against a $210 \mathrm{kDa}$ glycoprotein of the nuclear pore complex as a prognostic marker in patients with primary biliary cirrhosis. J Gastroenterol Hepatol 1998;13:257-65. 
17 Invernizzi P, Podda M, Battezzati PM, et al. Autoantibodies against nuclear pore complexes are associated with more active and severe liver disease in primary biliary cirrhosis. J Hepatol 2001;34:366-72

18 Muratori P, Muratori L, Ferrari R, et al. Characterization and clinical impact of antinuclear antibodies in primary biliary cirrhosis. Am J Gastroenterol 2003;98:431-7.

19 Metcalf $\mathrm{J}$, James $\mathrm{O}$. The geoepidemiology of primary biliary cirrhosis. Semin Liver Dis 1997; 17:13-22.

20 Kim WR, Lindor KD, Locke GR 3rd, et al. pidemiology and natural history of primary biliary cirrhosis in a US community. Gastroenterology 2000;1 19:1631-6.

21 Watson RG, Angus PW, Dewar M, et al. Low prevalence of primary biliary cirrhosis in Victoria, Australia. Melbourne Liver Group. Gut 1995:36:927-30.

22 Witt-Sullivan $\mathrm{H}$, Heathcote J, Cauch $\mathrm{K}$, et al. The demography of primary biliary cirrhosis in Ontario, Canada. Hepatology 1990;12:98-105.

23 Ludwig J, Dickson ER, McDonald GS. Staging of chronic nonsuppurative destructive cholangitis (syndrome of primary biliary cirrhosis). Virchows Arch A Pathol Anat Histol 1978;379:103-12.

24 Chazouilleres O, Wendum D, Serfaty L, et al. Primary biliary cirrhosisautoimmune hepatitis overlap syndrome: clinical features and response to therapy. Hepatology 1998;28:296-301.

25 Alvarez F, Berg PA, Bianchi FB, et al. International Autoimmune Hepatitis Group Report: review of criteria for diagnosis of autoimmune hepatitis. J Hepatol 1999;31:929-38.

26 Dickson ER, Grambsch PM, Fleming TR, et al. Prognosis in primary biliary cirrhosis: model for decision making. Hepatology 1989;10:1-7.

27 Dalekos GN, Wedemeyer H, Obermayer-Straub P, et al. Epitope mapping of cytochrome P4502D6 autoantigen in patients with chronic hepatitis $C$ during alpha-interferon treatment. J Hepatol 1999;30:366-75.

28 Dalekos GN, Makri E, Loges S, et al. Increased incidence of anti-LKM autoantibodies in a consecutive cohort of hepatitis $C$ patients from central Greece. Eur J Gastroenterol Hepatol 2002;14:35-42.

29 Dalekos GN, Zachou K, Liaskos C, et al. Autoantibodies and defined target autoantigens in autoimmune hepatitis: an overview. Eur J Intern Med 2002;13:293-303.
30 Bogdanos DP, Baum H, Sharma UC, et al. Antibodies against homologous microbial caseinolytic proteases $\mathrm{P}$ characterise primary biliary cirrhosis. J Hepatol 2002;36:14-21

31 Bogdanos DP, Baum H, Grasso A, et al. Microbial mimics are major targets of crossreactivity with human pyruvate dehydrogenase in primary biliary cirrhosis. J Hepatol 2004:40:31-9.

32 Grey HM, Kunkel HG. H chain subgroups of myeloma proteins and normal 7s gamma-globulin. J Exp Med 1964;120:253-66

33 Surh CD, Cooper AE, Coppel RL, et al. The predominance of $\lg G 3$ and $\lg M$ isotype antimitochondrial autoantibodies against recombinant fused mitochondrial polypeptide in patients with primary biliary cirrhosis. Hepatology 1988;8:290-5.

34 French MA, Bernstein RM. Immunoglobulin G subclass distribution of autoantibodies in systemic sclerosis, primary biliary cirrhosis, and overlap syndromes. Ann Rheum Dis 1987:46:436-40.

35 Miyachi K, Hankins RW, Matsushima H, et al. Profile and clinical significance of anti-nuclear envelope antibodies found in patients with primary biliary cirrhosis: a multicenter study. J Autoimmun 2003;20:247-54.

36 Bogdanos DP, Pares A, Rodes J, et al. Primary biliary cirrhosis specific antinuclear antibodies in patients from Spain. Am J Gastroenterol 2004;99:763-4.

37 Van Norstrand MD, Malinchoc M, Lindor KD, et al. Quantitative measurement of autoantibodies to recombinant mitochondrial antigens in patients with primary biliary cirrhosis: relationship of levels of autoantibodies to disease progression. Hepatology 1997;25:6-11.

38 Jefferis R. Structure-function relationships in human immunoglobulins. Neth J Med 1991;39:188-98.

39 Anderson CL, Looney RJ. Immunoglobulin G Fc receptors of human leukocytes. Methods Enzymol 1987;150:524-36.

40 Wiener $\mathbf{E}$, Jolliffe VM, Scott HC, et al. Differences between the activities of human monoclonal $\lg G 1$ and $\lg G 3$ anti-D antibodies of the Rh blood group system in their abilities to mediate effector functions of monocytes. Immunology 1988:65:159-63.

41 Vergani D, Mieli-Vergani G, Mondelli M, et al. Immunoglobulin on the surface of isolated hepatocytes is associated with antibody-dependent cell-mediated cytotoxicity and liver damage. Liver 1987;7:307-15. 